Z Gerontol Geriat 2017 · 50:97-98 DOI 10.1007/s00391-017-1182-x Online publiziert: 2. Februar 2017 (c) Springer-Verlag Berlin Heidelberg 2017

CrossMark

\section{Daniel Kopf ${ }^{1}$ ' Walter Hewer ${ }^{2}$}

${ }^{1}$ Kath. Marienkrankenhaus GmbH, Hamburg, Deutschland

${ }^{2}$ Klinik für Gerontopsychiatrie, Klinikum Christophsbad, Göppingen, Deutschland

\title{
Praktikable und wirksame Behandlungsansätze bei körperlicher und psychiatrischer Komorbidität
}

Geriatrische Patienten, die sowohl an körperlichen Störungen und Funktionseinschränkungen als auch an psychischen Störungen leiden, stellen Behandler im Alltag vor besondere Herausforderungen. Dies gilt gleichermaßen für älter werdende, chronisch psychisch kranke Menschen, bei denen im Alter körperliche Erkrankungen zunehmend relevant werden, wie für Menschen mit körperlicher Multimorbidität, bei denen im Alter erstmals psychische oder neuropsychiatrische Symptome auftreten.

Zum einen gibt es ein Ressourcenproblem: Diese Patienten benötigen deutlich mehr Zeit und Zuwendung, aber auch interdisziplinäres Fachwissen und Vernetzung, sodass in einer alternden Gesellschaft, deren Gesundheitswesen eine große Zahl von älteren Patienten in kurzer Zeit mit hoher Ergebnisqualität behandeln soll, oft die Ressourcen nicht auszureichen scheinen.

Zum anderen gibt es ein Evidenzproblem: Gängige Leitlinien fokussieren ihre Empfehlungen häufig auf Entscheidungsfelder, für die es belastbare Evidenz im Idealfall auf der Basis von mehreren randomisierten kontrollierten klinischen Studien gibt. Klinische Studien führen relevante Multimorbidität, z. B. mit Nierenfunktionsstörungen, häufig als Ausschlusskriterien und schließen selten Menschen im Alter über 80 Jahren ein. Folgerichtig bleiben Leitlinienempfehlungen oft vage.

Also ein unlösbares Problem? Keinesfalls! Vollmar et al. haben kürzlich ge- zeigt, dass ein interdisziplinärer Ansatz, wie er beispielsweise im „Aging Brain Care Program" in Indianapolis entwickelt wurde, auch in Deutschland realisierbar ist [7]. Zahlreiche Initiativen haben in den letzten Jahren im deutschen Sprachraum integrierte Behandlungseinrichtungen entwickelt, sei es in Form eines Liaisondienstes, einer internistischen Behandlung in gerontopsychiatrischen Einrichtungen oder in Spezialstationen für kognitiv eingeschränkte Menschen in geriatrischen Institutionen $[4,5]$.

Wie hoch wirksam integrative Behandlungskonzepte sind, hat erst kürzlich eine große Studie gezeigt, in der bei multimorbiden Patienten mit depressiven Störungen nicht nur die depressive Symptomatik, sondern sogar die Mortalität nachhaltig gesenkt werden konnte [3]. Allerdings reicht die einfache, vielleicht unkritische zusätzliche Verordnung eines Antidepressivums nicht aus. Eine solche Vorgehensweise bessert weder die körperliche Prognose noch die depressive Symptomatik in ausreichender Weise [2]. Ansätze, die psychopharmakologische Strategien mit psychotherapeutischen und Setting-basierten Ansätzen und dabei die besondere Vulnerabilität geriatrischer Patienten berücksichtigen, sind erforderlich [6].

Die Autoren dieses Hefts haben sich auf die Suche gemacht, nach ebenso evidenzbasierten und nachweislich wirksamen, wie auch im praktischen Alltag und in der klinischen Versorgungsrea- lität umsetzbaren Behandlungsansätzen. Dabei haben sie verfügbare Information aus klinischen Studien auf hohem methodischen Niveau und Empfehlungen von Leitlinien berücksichtigt. Wo aber der klinische Alltag Fragen stellt, die von solchen Empfehlungen nicht sicher beantwortet werden können, haben sie als weitere Erkenntnisquellen pathophysiologisch begründete Konzepte und klinische Erfahrung einfließen lassen. So gelangen sie zu fundierten und gleichermaßen praxistauglichen Behandlungsstrategien.

G. Eschweiler fasst ausgewählte neue Entwicklungen auf dem Gebiet der Altersdepression aus biopsychosozialer Perspektive zusammen. Die Übersicht unterstreicht die Notwendigkeit, auf den verschiedenen klinisch bedeutsamen Ebenen - Pathogenese, Diagnostik, Therapie etc. - bei jedem einzelnen Patienten eine Würdigung der individuell bedeutsamen biologischen und psychosozialen Aspekte vorzunehmen. Der Autor geht näher ein auf neuere psychotherapeutische Verfahren, die bekanntlich in den letzten Jahren stark an Bedeutung gewonnen haben. Schließlich arbeitet er heraus, dass innovative therapeutische Konzepte ohne ein besonderes Augenmerk auf die verfügbaren personellen Ressourcen und vorhandenen Versorgungsstrukturen nicht erfolgreich implementiert werden können.

Die Behandlung mit Psychopharmaka, speziell Antipsychotika, bei Alterspatienten mit Demenz und/oder Delir wird 
seit einigen Jahren wegen der damit verbundenen Risiken zunehmend kritisch gesehen. Gleichwohl ist ihre Anwendung im Versorgungsalltag eher die Regel denn die Ausnahme. W. Hewer und C. Thomas setzen sich mit diesem Spannungsfeld auseinander, unter Berücksichtigung des Handlungsdrucks, der aufgrund der mit Demenz und Delir assoziierten psychopathologischen Störungen regelmäßig entsteht. Auch wenn leitliniengestützte Aussagen nur eingeschränkt getroffen werden können, sind Psychopharmaka aus Sicht der Autoren bei sorgfältiger Indikationsstellung und adäquater Verlaufskontrolle ein unverzichtbarer Bestandteil multimodaler Therapiekonzepte für die betroffenen alten Menschen. Naturgemäß können sie aber individuell erforderliche medizinische und pflegerische Maßnahmen ebenso wenig ersetzen wie im multiprofessionellen Kontext durchgeführte nichtmedikamentöse Therapien.

Dass die längerfristige Verordnung von Benzodiazepinen generell und ganz besonders im Alter sehr kritisch zu bewerten ist, ist unumstritten. Daraus sollte jedoch nicht abgeleitet werden so eine der Kernbotschaften von D. Wolter -, dass ein generelles Ausschleichen bzw. Absetzen in jedem Fall sozusagen „im Schnellschuss“ erfolgen sollte. Er plädiert vielmehr für ein individualisiertes Vorgehen und gibt konkrete Empfehlungen, wie bei der Dosisreduktion und dem Ausschleichen vorzugehen ist. Diese Ziele können nicht ohne eine angemessene Einbeziehung der Patientenperspektive erreicht werden, ein Prinzip, dessen Bedeutung für die (Alters)Medizin zunehmend erkannt wird (American Geriatrics Society Expert Panel on the Care of Older Adults with Multimorbidity [1]).

Ergänzt werden diese drei Übersichten durch eine gerontopsychologische Originalarbeit von Fankhauser et al., in der bei 189 älteren Personen, davon 71 mit kognitiven Störungen, Wechselbeziehungen zwischen kognitiver Kompetenz und sozialer Einbindung untersucht wurden. Die Autoren belegen mit ihren Daten, dass Selbstwirksamkeit hierbei eine relevante Mediatorvariable darstellt. Da Selbstwirksamkeit durch Interventio- nen beeinflusst werden kann, liegt damit die Frage nahe, welche präventiven und therapeutischen Potenziale für die kognitive Gesundheit durch einen solchen Ansatz realisiert werden können.

Wir wünschen uns, dass das vorliegende Heft der Zeitschrift für Gerontologie und Geriatrie dazu beiträgt, Behandlern alltagstaugliche Optionen auf fundierter wissenschaftlicher Basis für die Behandlung dieser manchmal besonders belastenden, aber immer belasteten Gruppe von Patienten nahezubringen.

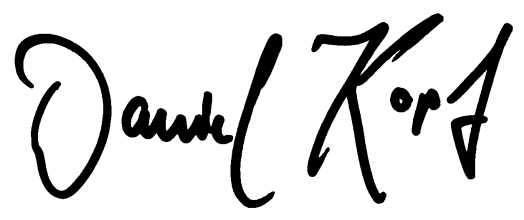

Daniel Kopf

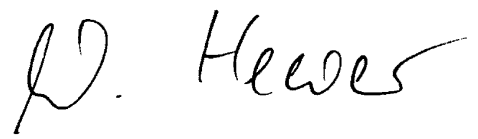

Walter Hewer

\section{Korrespondenzadresse}

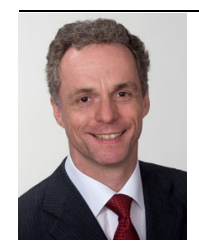

PD Dr. med. D. Kopf

Kath. Marienkrankenhaus $\mathrm{GmbH}$

Alfredstr. 9, 22087 Hamburg,

Deutschland

kopf.geriatrie@

marienkrankenhaus.org

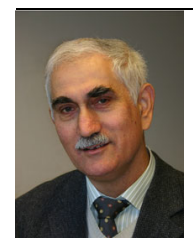

Prof. Dr. med. W. Hewer

Klinik für Gerontopsychiatrie, Klinikum Christophsbad Faurndauer Str. 6-28, 73035 Göppingen,

Deutschland

walter.hewer@

christophsbad.de

Interessenkonflikt. D. Kopf und W. Hewer geben an, dass kein Interessenkonflikt besteht.

\section{Literatur}

1. American Geriatrics Society Expert Panel on the Care of Older Adults with Multimorbidity (2012) Guiding principles for the care of older adults with multimorbidity: an approach for clinicians. J Am Geriatr Soc 60:E1-E25

2. Angermann $C E$, Gelbrich $G$, Störk $S$, Gunold $H$, Edelmann F, Wachter R, Schunkert $H$, Graf $T$, Kindermann I, Haass M, Blankenberg S, Pankuweit S, Prettin C, Gottwik M, Böhm M, Faller H, Deckert J, ErtI G, MOOD-HF Study Investigators Committee Members(2016) Effect of escitalopram onall-cause mortality and hospitalization in patients with heart failure and depression: the MOOD-HF randomized clinical trial. JAMA 315(24):2683-2693

3. Gallo JJ, Hwang S, Joo JH, Bogner HR, Morales KH, Bruce ML, Reynolds CF 3rd (2016) Multimorbidity, depression, and mortality in primary care: randomized clinical trial of an evidence-based depression care management program on mortality risk. J Gen Intern Med 31 (4):380-386

4. Hewer W, Stark HW (2010) General medical interventions in patients with dementia treated in a psychogeriatric unit. Z Gerontol Geriatr 43(3):180-182

5. Hofmann W, Rösler A, Vogel W, Nehen HG (2014) Special care units for acutely ill patients with cognitive impairment in Germany. Position paper. ZGerontol Geriatr 47(2):136-140

6. Hummel J, Weisbrod C, Boesch L, Himpler K, Hauer K, Hautzinger M, Gaebel A, Zieschang T, Fickelscherer A, Diener S, Dutzi I, Krumm B, Oster P, Kopf D (2016) AIDE-acute illness and depression in elderly patients. Cognitive behavioral group psychotherapy in geriatric patients with comorbid depression: a randomized, controlled trial. J Am Med Dir Assoc. doi:10.1016/j.jamda.2016.10.009

7. Vollmar HC, Thyrian JR, LaMantia MA, Alder CA, Guerriero Austrom MM, Callahan C, Leve V, Hoffmann W, Boustani M (2016) Aging brain care program from indianapolis: transferability to the German healthcare system. Z Gerontol Geriatr 49(1):32-36 